\title{
High-speed 1D Raman analyzer for temperature and major species measurements in combustion environment
}

\author{
ChaObo Yang ${ }^{1}$, Hao TANG ${ }^{1}$, and Gaetano Magnotti ${ }^{1, *}$ \\ ${ }^{1}$ Clean Combustion Research Center (CCRC), King Abdullah University of Science and Technology (KAUST), Thuwal, Saudi Arabia 23955-6900 \\ *Corresponding author: gaetano.magnotti@kaust.edu.sa
}

Compiled April 9, 2020

In this paper, we demonstrate a $5 \mathrm{kHz}$ 1D Raman instrument for temporally and spatially resolved, quantitative measurements of temperature and major species $\left(\mathrm{N}_{2}, \mathrm{O}_{2}, \mathrm{H}_{2}\right.$, and $\left.\mathrm{H}_{2} \mathrm{O}\right)$ concentration in $\mathrm{H}_{2}$-air flames. The major constituent of the system are a pulse-burst laser operated at $5 \mathrm{kHz}$, and four back-illuminated CCD cameras operated in subframe burst-gating mode. The use of CCD cameras allows achieving highsampling rate with no compromise in instrument precision, but it requires one camera for each species of interest. A cascade of dichroic mirrors and bandpass filters spectrally separates the Raman signal associated to each of the four species, and direct it to a separate camera. Measurements in a well characterized $\mathrm{H}_{2}$-air premixed flat flame, shows that the system has comparable precision with the low speed Raman system. The measuring uncertainty of species mole fraction ranges between $1 \%\left(\mathrm{~N}_{2}\right)$ to $3 \sim 4 \%\left(\mathrm{O}_{2}\right.$ in lean flames). Measurements in laminar and turbulent $\mathrm{H}_{2} / \mathrm{N}_{2}$ jet flames show good agreement with the theoretical prediction. By measuring all species simultaneously important combustion quantities such as the mixture fraction are also derived.

(C) 2020 Optical Society of America

http://dx.doi.org/10.1364/ao.XX.XXXXXX

In recent years, Raman scattering has been widely applied as a non-intrusive combustion diagnostics technique. It can provide highly precise and accurate measurements of major species concentrations and temperature in non-sooting flames[13]. Because of the small Raman scattering cross-section, lasers providing several hundreds of $\mathrm{mJ} /$ pulse are required for measurements in reacting flows. Limited by the thermal load of the conventional solid-state lasers, and by the long readout time of low-noise CCD cameras, the sampling rates of Raman combus- tion diagnostics are typically limited to $5-10 \mathrm{~Hz}[4]$. However, high-speed measurements of temperature and species concentration are highly desirable to improve understanding of turbulent combustion dynamics, and to investigate combustion phenomena that last only a few ms. Recently, pulse-burst lasers delivering short (10 ms typically) bursts of high energy (hundreds of $\mathrm{mJ}$ ) laser pulses at $\mathrm{kHz}$ rates [5, 6], have extended Raman scattering combustion measurements to high sampling rates. High-speed 1D Raman measurements have been demonstrated both in nonreactive[7] and reactive flows[8], and more recently extended to 2D imaging for non-reacting flows at elevated pressures[9].

Raman instruments for combustion diagnostics typically employ a fast gate (order of $\mu \mathrm{s}$ ) to suppress contributions from flame luminosity, which can lead to inaccuracies, and lower the signal to noise ratio (SNR). State-of-the-art, low speed, Raman instruments usually rely on low-noise back-illuminated CCD cameras, coupled to a fast mechanical shutter. Superior SNR and a few $\mu$ s gating time can be achieved by this setup, but the repetition rate of the rotating wheels based shutter is limited to frequencies lower than $30 \mathrm{~Hz}[10,11]$. To date, all the highspeed Raman instruments for combustion diagnostics employed high-speed CMOS cameras, coupled to a high-speed intensifier for fast gating. High-speed CMOS cameras offer higher acquisition rates, but inferior readout noise, quantum efficiency, and dynamic range than low-noise back-illuminated CCD cameras. An image intensifier can provide fast optical gating (down to ns) but is not the preferred option for low speed Raman based quantitative measurements because of losses in the image quality, spatial and spectral resolution, and dynamic range.

Subframe burst imaging $[12,13]$ takes advantage of the rapid vertical shift $(1.2 \mu \mathrm{s} /$ row $)$ of frame transfer CCD sensors to achieve short exposure time and $\mathrm{kHz}$ sampling rates while maintaining the low readout noise characteristic of the CCD sensor. In this mode of operation, only the bottom $\mathrm{N}$-rows of the sensor are illuminated and, after each laser pulse, the charge is shifted to a masked region rather than to the serial register for readout. Once the sensor (including the frame transfer area) is fully filled, readout, and analog to digital conversion is conducted slowly $(100 \mathrm{kHz})$ to minimize the readout noise. For a frame transfer camera with $1024 \times 1024$ pixels sensor, and an equally large frame-transfer area, by exposing 20 rows, 102 frames can be acquired. This is a major drawback using high-speed lasers 
which can operate continuously, but it is irrelevant when using a pulse burst laser, that can only provide a limited train of pulses. Subframe burst imaging allows combining high-sampling rates to the superior noise characteristic offered by back-illuminated CCD cameras. This readout mode was combined to pulse-burst lasers for $100 \mathrm{kHz}$ 0D ps-CARS thermometry[14] but with a precision ranging from $6 \%$ to $9 \%$. The approach was then employed in 1D, $10 \mathrm{kHz}$ Rayleigh/Raman measurements of temperature and methane concentration[15] achieving $100 \mu \mathrm{m}$ spatial resolution and temperature precision $<0.7 \%$. By exposing only 25 pixel rows, spectral dispersion along the vertical (rows) direction is impossible, limiting Raman measurements to a single species. Viceversa, by dispersing the Raman signal along the horizontal direction (1024 columns), subframe burst imaging can be applied to 0D or quasi 1D Raman measurements of all major species(as discussed in[16]).

In this letter, for the first time, we applied subframe burst imaging to high-speed 1D Raman measurements of all major species $\left(\mathrm{H}_{2}, \mathrm{O}_{2}, \mathrm{~N}_{2}, \mathrm{H}_{2} \mathrm{O}\right)$ in $\mathrm{H}_{2}$-air flames. This is a major development from previous work, because simultaneous measurements of all major species allow deriving several critical combustion quantities, such as temperature, mixture fraction, progress variables, and atom ratios, resulting in a much greater insight than what can be provided by any other high-speed technique, generally limited to a few species.

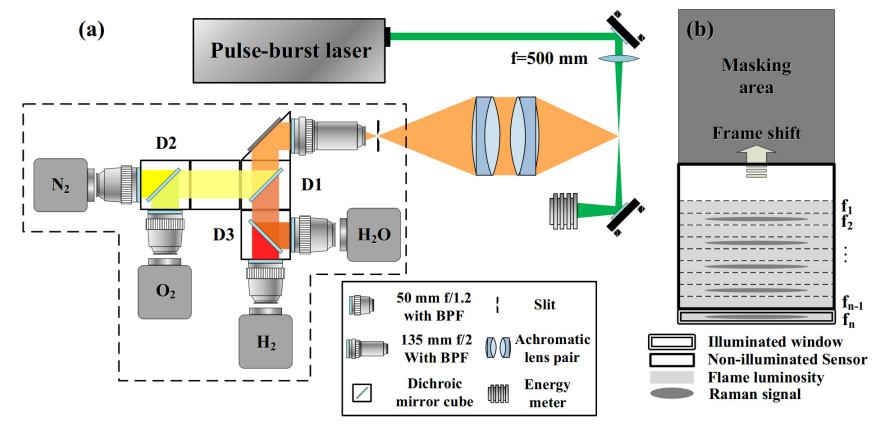

Fig. 1. (a) Experimental setup of the H1DRA system, (b) Working flow of the subframe burst imaging

Figure 1 shows the experimental setup of the high-speed multi-species 1D Raman analyzer (H1DRA) and the schematic diagram for subframe burst gating imaging employed in this work. A pulse-burst laser (Spectral Energies, Quasimodo) operated at $5 \mathrm{kHz}$ repetition rate, with a burst duration of $10 \mathrm{~ms}$, provides the high-speed laser train at $532 \mathrm{~nm}$ used as the excitation source. A $500 \mathrm{~mm}$ focal lens, focused the laser beams to a spot size of $\sim 140 \mu \mathrm{m}$ (FWHM) at the measurement volume. Benefiting from the acousto-optic modulator (AOM) based seed pulse generator, the laser pulse duration can be varied continuously from 10 to 1000 ns (FWHM)[9]. Longer pulses allow to deposit more energy at the measurement volume without incurring in optical breakdown, but the conversion efficiency of the second harmonic generator drops with the pulse length. In this configuration ( $5 \mathrm{kHz}, \sim 140 \mu \mathrm{m}$ spot size) we determined that a pulse duration of 130 ns maximizes the pulse energy ( $\sim 450 \mathrm{~mJ} /$ pulse) and avoid the laser induced breakdown. The diverging beam after the focal point is reflected to a pyroelectric energy-meter (Coherent, J-50MT-10KHZ) to monitor the energy fluctuation on a shot-to-shot basis. A pair of achromatic lens $\operatorname{collects}(\Phi=100 \mathrm{~mm}, \mathrm{f} / 3)$ and refocus $(\Phi=100 \mathrm{~mm}, \mathrm{f} / 4)$ the Raman scattering signal. A slit, placed at the focal plane, acts as a spatial filter for the subframe imaging. The Raman signal is then collimated by a telephoto lens (Canon, $135 \mathrm{~mm} \mathrm{f/2}$ ) and filtered by a bandpass filter (Semrock, FF01-565/133) to block the Rayleigh scattering and surface scattering. The roughly collimated Raman signal then enters the wavelength separation unit, a cascade of dichroic mirrors (Semrock, D1: FF640, D2: FF580, D3:FF662) that spectrally separates the Raman contributions associated to the four species probed. Each of the four resulting Raman "beams" passes through an opportunely chosen bandpass filter (Semrock, $\mathrm{H}_{2} \mathrm{O}$ : FF01-655/15, $\mathrm{H}_{2}$ : FF01-680/13, $\mathrm{N}_{2}$ : FF01-605/15, O 2 : FF01-575/15) to narrow the transmission spectral window and further reduce interference of stray light. Four off-the-shelf lenses (Nikon, $50 \mathrm{~mm} \mathrm{f/1.2)} \mathrm{focused} \mathrm{the} \mathrm{Raman}$ signal onto four back-illuminated EMCCD cameras (Princeton Instruments, ProEM-HS), one for each species of interest. The overall magnification of the collection optics is $\sim 0.5$ and the pixel resolution is $26 \mu \mathrm{m}$. The measurement volume is a cylinder of $140 \mu \mathrm{m}$ of cross section (determined by the beam spot size) and $\sim 60 \mu \mathrm{m}$ long (estimated from a resolution test target)

All the lenses, mirrors and filters are mounted in a Thorlabs $60 \mathrm{~mm}$ cage system to simplify the alignment, ensure a common optical axis. Under the subframe burst mode, the Raman signal has to be imaged on the bottom of the sensor to maximize the record length within one readout cycle. The cameras were detached from the cage system, and mounted on a 2-axis translation stage for focusing, and adjusting the camera height. This approach allows illuminating the bottom rows of the sensor, while keeping the Raman signal along the lens optical axis. Care was taken in enclosing the wavelength separation unit, and all the cameras, to minimize stray light contributions. Careful registration of the four cameras is essential to obtain accurate results. The cameras were properly aligned, so that a $50 \mu \mathrm{m}$ pinhole placed at the center of measurement volume was imaged on the same location on all four sensors. Images of a target with $0.5 \mathrm{~mm}$ spacing mesh grid provide a reference for spatial registration of the cameras for correcting small differences in the magnification.

The working flow of the subframe burst imaging is illustrated in Fig. 1(b) and details can be found in ref.[15]. In this work, the subframe imaging size was set to $24 \times 1024$ pixels resulting in 85 subframes per readout cycle. The spatial filtering silt was set to $\sim 545 \mu \mathrm{m}$ (21 pixels), slightly narrower than the exposed region on the camera, to avoid the inter-subframe crosstalk, but larger than the Raman signal image size (350-400 $\mu \mathrm{m})$. Subframe burst imaging also provides fast gating capability to suppress flame luminosity. By operating the laser at $5 \mathrm{kHz}$, and the camera at $10 \mathrm{kHz}$, two frames, each with $100 \mu$ s exposure were collected for each laser pulse. One frame would be synchronized with the laser and contains the Raman signal and the flame luminosity, the other only the flame luminosity, providing instantaneous background correction.

Operating with CCD cameras allows direct implementation of noise reduction strategies developed for low speed Raman instruments. In particular, hardware binning is performed in the vertical direction and the resulting $1 \mathrm{D}$ profiles for each species are denoised using the wavelet adaptive thresholding and reconstruction algorithm (WATR) [17], routinely used for $10 \mathrm{~Hz}$ Raman measurements of spatial gradients. Threshold levels for the WATR algorithm are based on the local signal level, and on the gain and RMS of the camera readout noise, with no user-tunable parameters. The algorithm has been extensively validated for $10 \mathrm{~Hz}$ 1D Raman measurements, and it has been demonstrated that it increases the signal-to-noise ratio, without introducing artificial smoothing[17].The denoised profiles are 
then background subtracted and energy normalized to remove the effect of pulse energy fluctuations. Spatial throughput correction curves are obtained for all species from measurements in uniform non-reacting mixtures for $\mathrm{N}_{2}, \mathrm{O}_{2}$, and $\mathrm{H}_{2}$, and in the product region of a flat flame for $\mathrm{H}_{2} \mathrm{O}$. Thermal lensing effect (thermal induced refractive index gradient inside the laser crystal) causes changes in the focusing of the laser beam. The effect is very repeatable from burst-to-burst, and it is accounted for by using throughput curves that are also function of the pulse index within a burst.

The resulting 1D Raman data were then evaluated following the "hybrid method" [18]. For each spatial location x, we obtained the column vector $S(x)$ containing the pre-processed signal associated to each species/camera, which is related to the unknown species number densities $N(x)$ vector through the linear equation:

$$
S(x)=C(T(x)) N(x)
$$

In the $C$ matrix, the diagonal elements are the Raman responses of the various species, whereas off-diagonal elements $C_{i j}$ represent crosstalks between Raman channels, that are contributions from species $j$ to species $i$. The matrix is sparse, and the only significant contributions in $\mathrm{H}_{2}$-air flame are crosstalks from $\mathrm{H}_{2}$ to $\mathrm{O}_{2}$ and $\mathrm{H}_{2} \mathrm{O}$. The temperature dependencies of the $\mathrm{C}$ matrix terms are obtained as follows: 1 ) theoretical spectral libraries (RAMSES [19]) are obtained for the four species of interest and for temperature varying from $250 \mathrm{~K}$ to $2500 \mathrm{~K}$; 2) the spectra are multiplied by the experimental transmission spectra obtained for each species channel, and integrated over the entire wavelength range. Thanks to the absence of spectral broadening and bowing effect introduced by a grating, the H1DRA has crosstalk similar to what observed in [18] despite the softer spectral separation of a dichroic/filter combination.

Although the temperature dependency is computed from theoretical spectra, a calibration is needed to obtain their absolute values (function of the overall collection system). Measurements in ambient temperature dry air and $\mathrm{H}_{2}$ flow provided the Raman response of $\mathrm{N}_{2}, \mathrm{O}_{2}$, and $\mathrm{H}_{2}$. Measurements in a reference $\mathrm{H}_{2}$-air flat premixed flame (Mckenna burner, $\mathrm{H}_{2}$ 4.88 SLPM, $\Phi$ $=1.3$ )[20] provided the Raman response of $\mathrm{H}_{2} \mathrm{O}$ and cross-talk from $\mathrm{H}_{2}$ to $\mathrm{O}_{2}$ and $\mathrm{H}_{2} \mathrm{O}$. The data reduction is iterative. An initial temperature is guessed, and initial composition is computed from Eq.1. Ideal gas law provides an updated temperature, and the process is iterated until the updated temperature is within 5 $\mathrm{K}$ of the previous value.

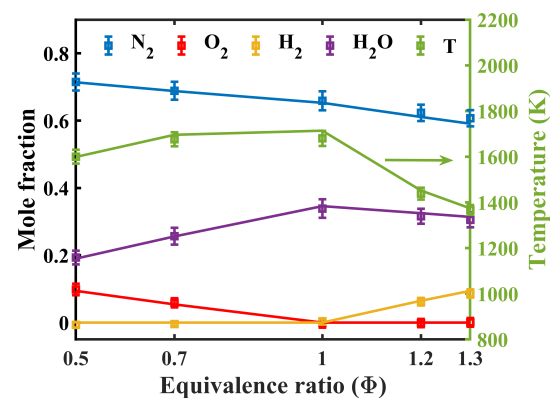

Fig. 2. Mole fraction (left) and temperature (right) measurements in the $\mathrm{H}_{2}$ premixed flat flames $(\Phi=0.5 \sim 1.3)$ ).

To evaluate the precision (standard deviation/mean value) and relative accuracy (deviation between mean value and reference data) of the H1DRA, we conducted benchmark tests in a series of reference $\mathrm{H}_{2}$-air flat premixed flames. The $\mathrm{H}_{2}$ flow rate was kept at 9.85 SLPM, and 5 different equivalence ratios between 0.5 and 1.3 were measured. We carefully duplicated the configuration in Ref.[20] including coolant temperature, and the measuring location to minimize discrepancies. Product temperatures ( CARS) and mole fractions (chemical equilibrium analysis at measured temperature) are tabulated in Ref. [20]. For each equivalence ratio, a total of 1260 frames were collected from 30 consecutive bursts. The average major species mole fraction $(X)$ and temperature for all the tested cases are shown in Fig. 2. Standard deviations divided by the mean value are used as indication of the instrument precision. For $\Phi=1.3$ and $T=1374 \mathrm{~K}$, the precision of mole fraction measurements of $\mathrm{N}_{2}, \mathrm{H}_{2}$, and $\mathrm{H}_{2} \mathrm{O}$ is $0.7 \%, 2.8 \%$ and $2.3 \%$, respectively, and for the temperature is $1.7 \%$. For $\Phi=0.7$ and $T=1696 \mathrm{~K}$ the precision for $\mathrm{N}_{2}, \mathrm{O}_{2}$, and $\mathrm{H}_{2} \mathrm{O}$ is $0.8 \%, 3.5 \%$ and $1.8 \%$, and for the temperature is $2.0 \%$. Error bars in Fig. 2 represent \pm 5 standard deviations for the mole fraction measurements and \pm 1 standard deviation for the temperature. Measurements' precision does not change significantly within the $6 \mathrm{~mm}$ probe volume. Species mole fraction precision are comparable with what reported for conventional $5 \mathrm{~Hz} 1 \mathrm{D}$ Raman system. Temperature precision can be greatly improved by adding one additional camera for Rayleigh scattering. The relative accuracy, (evaluated based on Ref. [20]) is within $48 \mathrm{~K}$ for temperature and 0.01 for mole fractions measurements.
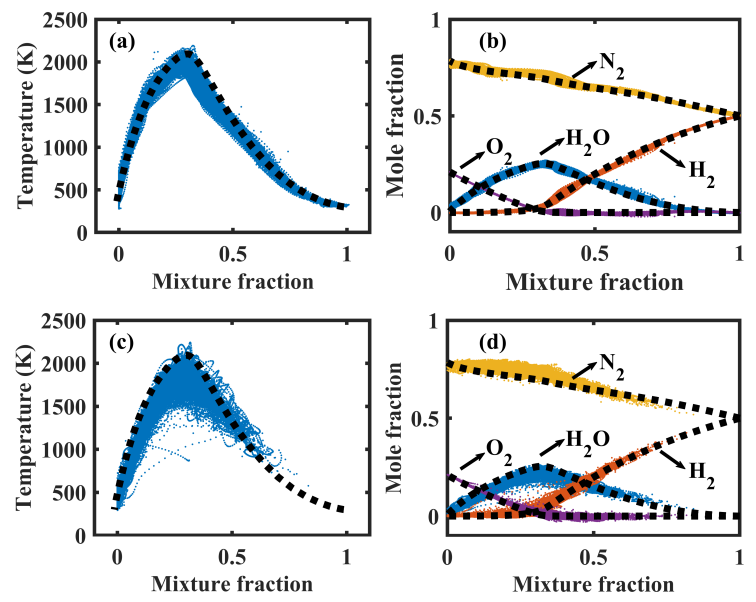

Fig. 3. Mole fraction and temperature versus mixture fraction for the laminar $(\mathrm{a}, \mathrm{b})$ and turbulent $(\mathrm{c}, \mathrm{d}) \mathrm{H}_{2} / \mathrm{N}_{2}$ jet flames

Figure 3 summarizes results taken in a laminar and in a turbulent $\mathrm{H}_{2} / \mathrm{N}_{2}$ jet diffusion flame. The main components of the diffusion flame burner are two concentric stainless steel tubes. The inner tube (ID $4.5 \mathrm{~mm}$, OD $6.5 \mathrm{~mm}$ ) supplies the fuel stream, and the outer tube (ID $10.5 \mathrm{~mm}$, OD $12.5 \mathrm{~mm}$ ) supplied co-flow air. For the laminar case, the fuel stream was a mixture of $\mathrm{H}_{2}$ $50 \% / \mathrm{N}_{2} 50 \%$ jet with a $\mathrm{Re} \approx 1180$. Raman measurements were taken at an axial position of $x / D=2$. In the turbulent case, the total flow rate of the $\mathrm{H}_{2} 50 \% / \mathrm{N}_{2} 50 \%$ jet was increased to $\operatorname{Re} \approx 19800$, and measurements were taken at $x / D=15$. Thirty bursts of data were acquired for both cases. The measured temperature and species mole fraction are plotted versus the local Bilger mixture fraction $(Z)$ and presented in Fig. 3. The Bilger mixture fraction[21] is defined as:

$$
Z=\frac{v Y_{H}-Y_{O}+Y_{O, 2}}{v Y_{H, 1}+Y_{O, 2}}
$$


where $v$ is the stoichiometric mass ratio, $\left(Y_{H}, Y_{O}\right)$ the local mass fraction of atomic hydrogen and oxygen, $\left(Y_{H, 1}, Y_{O, 2}\right)$ the mass fraction atomic hydrogen and oxygen in the fuel and oxidizer stream.

Measurements from the laminar case are shown in Fig.3(a) and (b). Results from opposed flow simulation in Cantera with the GRI-Mech 3.0 mechanism and a strain rate of $50 \mathrm{~s}^{-1}$ are plotted as dashed black line to provide a qualitative comparison to the measurements. No obvious artifacts associated to errors in camera registration appear in these plots of mole fractions versus mixture fraction. The temperature given by the H1DRA system is obtained from the measurement of total number density. The slightly deviation of temperature (3\% to $5 \%$ ) shown in the flame front region can be attributed to the degradation of signal to noise ratio caused by the lower total number density. As stated above, the instrument temperature precision can be improved by adding Rayleigh scattering channel. The turbulent case results were plotted in Fig.3(c) and (d). The temperature versus mixture fraction profile highlights that the turbulent flame is burning in non-premixed mode. Data points with temperature lower than the extinction limit of steady laminar flames suggest evidence of localized extinction. This behavior is clearly mirrored in the distribution of $\mathrm{H}_{2}$ and $\mathrm{O}_{2}$ mole fractions.

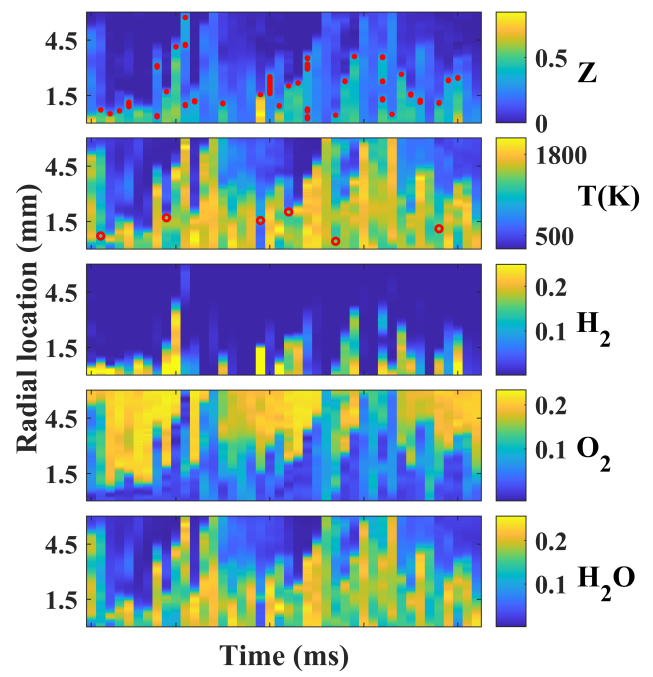

Fig. 4. Spatio-temporal resolved temperature, mole fraction and mixture fraction within one burst $(\sim 8 \mathrm{~ms})$

The H1DRA system can also provide time-resolved temporal evolution of temperature and species in the turbulent flame. It can offer more information for the research of turbulentcombustion dynamics such as the analysis of the local extinction of the flame. The spatial-temporal resolved information of $(T$, $X$, and $Z$ ) obtained within one burst is shown in Fig. 4. The locations with stoichiometric mixture fraction $\left(0.29<Z_{s t}<0.31\right)$ are highlighted with red dot in the time-resolved mixture fraction data. The absence of $Z_{s t}$ region in some pulses is caused by the movement of $Z_{s t}$ region out of the measurement volume. The temperature of stoichiometric mixture fraction region $\left(T_{s t}\right)$ can also be tracked to indicate the local extinction events. The location with $T_{s t}$ lower than the $70 \%$ of adiabatic temperature are labeled by circles in the temperature profile sequence to show the local extinction. However, higher temporal resolution are still required to portray these dynamics with more details.

In summary, we demonstrated a high-speed 1D Raman analyzer (H1DRA) and its application in the combustion diagnostics. To the best of our knowledge, this is the first reported high-speed Raman scattering measuring system based on the subframe burst mode of CCD camera with the ability of multispecies measurement and 1D spatial resolution. Combining with the pulse-burst laser, the system can provide multi-kilo-hertz repetition rate of measurements for major species concentrations and temperature. By avoiding instrument precision losses introduced by high-speed CMOS sensor and image intensifier, the H1DRA system has comparable measuring precision and accuracy with the widely used low speed Raman system, as shown by validation in $\mathrm{H}_{2}$-air premixed flat flames. Although demonstrated in steady turbulent flames, the instrument is especially suitable for the study of fast $(<10 \mathrm{~ms})$ combustion dynamic events, where a low-sampling rate system is inadequate. Thanks to a modular design and crosstalk-contributions comparable to those of conventional grating based system, the instrument can be extended to measure all 7 major species in $\mathrm{CH}_{4}$-air flames.

\section{FUNDING}

The research reported in this publication was supported by funding from King Abdullah University of Science and Technology (KAUST).

\section{REFERENCES}

1. R. Barlow and J. Frank, Symp. (International) on Combust. 27, 1087 (1998).

2. W. Meier, R. Barlow, Y. L. Chen, and J. Y. Chen, Combust. Flame 123, 326 (2000).

3. D. Geyer, A. Kempf, A. Dreizler, and J. Janicka, Proc. Combust. Inst. 30, 681 (2005).

4. R. S. Barlow, Proc. Combust. Inst. 31, 49 (2007).

5. M. N. Slipchenko, J. D. Miller, S. Roy, J. R. Gord, S. A. Danczyk, and T. R. Meyer, Opt. Lett. 37, 1346 (2012).

6. F. Fuest, M. J. Papageorge, W. Lempert, and J. A. Sutton, Opt. Lett. 37, 3231 (2012).

7. K. N. Gabet Hoffmeister, N. Jiang, W. Lempert, and J. Sutton, Appl. Phys. B 101, 1 (2010).

8. K. N. Gabet Hoffmeister, F. Fuest, and J. A. Sutton, 53rd AIAA Aerosp. Sci. Meet. p. 0531 (2015).

9. N. Jiang, P. S. Hsu, J. G. Mance, Y. Wu, M. Gragston, Z. Zhang, J. D. Miller, J. R. Gord, and S. Roy, Opt. letters 42, 3678 (2017).

10. P. Miles and R. Barlow, Meas. Sci. Technol. 11, 392 (2000).

11. Q.-V. Nguyen, "High-speed electromechanical shutter for imaging spectrographs," (2005). US Patent 6,937,331.

12. M. B. Sinclair, D. M. Haaland, J. A. Timlin, and H. D. Jones, Appl. optics 45, 6283 (2006).

13. J. Kojima, D. Fischer, and Q.-V. Nguyen, Opt. Lett. 35, 1323 (2010).

14. S. Roy, P. S. Hsu, N. Jiang, M. N. Slipchenko, and J. R. Gord, Opt. Lett. 40, 5125 (2015).

15. Y. Krishna, H. Tang, A. M. Elbaz, and G. Magnotti, Opt. Lett. 44, 4091 (2019).

16. G. Magnotti and Y. Krishna, Digit. Hologr. Three-Dimensional Imaging pp. JTu4A-17 (2018).

17. M. S. Sweeney, S. Hochgreb, M. J. Dunn, and R. S. Barlow, Combust. Flame 160, 322 (2013).

18. F. Fuest, R. Barlow, D. Geyer, F. Seffrin, and A. Dreizler, Proc. Combust. Inst. 33, 815 (2011).

19. D. Geyer, "1D-Raman/Rayleigh experiments in a turbulent opposed-jet," Ph.D. thesis, Technische Univ. Darmstadt (2005).

20. S. Prucker, W. Meier, and W. Stricker, Rev. Sci. Instruments 65, 2908 (1994).

21. R. Bilger, S. Stårner, and R. Kee, Combust. Flame 80, 135 (1990). 


\section{FULL REFERENCES}

1. R. Barlow and J. Frank, "Effects of turbulence on species mass fractions in methane/air jet flames," Symp. (International) on Combust. 27, 1087 - 1095 (1998).

2. W. Meier, R. Barlow, Y. L. Chen, and J. Y. Chen, "Raman/Rayleigh/LIF measurements in a turbulent $\mathrm{CH}_{4} / \mathrm{H}_{2} / \mathrm{N}_{2}$ jet diffusion flame: experimental techniques and turbulence-chemistry interaction," Combust. Flame 123, 326-343 (2000).

3. D. Geyer, A. Kempf, A. Dreizler, and J. Janicka, "Scalar dissipation rates in isothermal and reactive turbulent opposed-jets: 1-D-Raman/Rayleigh experiments supported by LES," Proc. Combust. Inst. 30, 681-689 (2005).

4. R. S. Barlow, "Laser diagnostics and their interplay with computations to understand turbulent combustion," Proc. Combust. Inst. 31, 49-75 (2007).

5. M. N. Slipchenko, J. D. Miller, S. Roy, J. R. Gord, S. A. Danczyk, and T. R. Meyer, "Quasi-continuous burst-mode laser for high-speed planar imaging," Opt. Lett. 37, 1346-1348 (2012).

6. F. Fuest, M. J. Papageorge, W. Lempert, and J. A. Sutton, "Ultrahigh laser pulse energy and power generation at $10 \mathrm{kHz}$," Opt. Lett. 37, 3231-3233 (2012).

7. K. N. Gabet Hoffmeister, N. Jiang, W. Lempert, and J. Sutton, "Demonstration of high-speed 1D Raman scattering line imaging," Appl. Phys. B 101, 1-5 (2010).

8. K. N. Gabet Hoffmeister, F. Fuest, and J. A. Sutton, "High-Speed 1D Raman/Rayleigh Scattering Imaging in Turbulent $\mathrm{H}_{2} / \mathrm{N}_{2}$ Flames," $53 \mathrm{rd}$ AIAA Aerosp. Sci. Meet. p. 0531 (2015).

9. N. Jiang, P. S. Hsu, J. G. Mance, Y. Wu, M. Gragston, Z. Zhang, J. D. Miller, J. R. Gord, and S. Roy, "High-speed 2D Raman imaging at elevated pressures," Opt. letters 42, 3678-3681 (2017).

10. P. Miles and R. Barlow, "A fast mechanical shutter for spectroscopic applications," Meas. Sci. Technol. 11, 392 (2000).

11. Q.-V. Nguyen, "High-speed electromechanical shutter for imaging spectrographs," (2005). US Patent 6,937,331.

12. M. B. Sinclair, D. M. Haaland, J. A. Timlin, and H. D. Jones, "Hyperspectral confocal microscope," Appl. optics 45, 6283-6291 (2006).

13. J. Kojima, D. Fischer, and Q.-V. Nguyen, "Subframe burst gating for Raman spectroscopy in combustion," Opt. Lett. 35, 1323-1325 (2010).

14. S. Roy, P. S. Hsu, N. Jiang, M. N. Slipchenko, and J. R. Gord, "100-kHzrate gas-phase thermometry using 100 -ps pulses from a burst-mode laser," Opt. Lett. 40, 5125-5128 (2015).

15. Y. Krishna, H. Tang, A. M. Elbaz, and G. Magnotti, "High-speed Rayleigh-Raman measurements with subframe burst gating," Opt. Lett. 44, 4091-4094 (2019).

16. G. Magnotti and Y. Krishna, "Quasi-1D High-Speed Raman/Filtered Rayleigh Scattering for Combustion Dynamics Applications," Digit. Hologr. Three-Dimensional Imaging pp. JTu4A-17 (2018).

17. M. S. Sweeney, S. Hochgreb, M. J. Dunn, and R. S. Barlow, "Multiply conditioned analyses of stratification in highly swirling methane/air flames," Combust. Flame 160, 322-334 (2013).

18. F. Fuest, R. Barlow, D. Geyer, F. Seffrin, and A. Dreizler, "A hybrid method for data evaluation in 1-D Raman spectroscopy," Proc. Combust. Inst. 33, 815-822 (2011).

19. D. Geyer, "1D-Raman/Rayleigh experiments in a turbulent opposed-jet," Ph.D. thesis, Technische Univ. Darmstadt (2005).

20. S. Prucker, W. Meier, and W. Stricker, "A flat flame burner as calibration source for combustion research: Temperatures and species concentrations of premixed $\mathrm{H}_{2}$ /air flames," Rev. Sci. Instruments 65, 2908-2911 (1994).

21. R. Bilger, S. Stårner, and R. Kee, "On reduced mechanisms for methane-air combustion in nonpremixed flames," Combust. Flame 80, 135 - 149 (1990). 